\title{
Non-linear radiative models of post-AGB stars: Application to HD 56126
}

\author{
A. B. Fokin ${ }^{1,2}$, A. Lèbre ${ }^{2}$, H. Le Coroller ${ }^{3}$, and D. Gillet ${ }^{3}$ \\ 1 Institute of Astronomy of the Russian Academy of Sciences, 48 Pjatnitskaya Str., Moscow 109017, Russia \\ 2 Groupe de Recherche en Astronomie et Astrophysique du Languedoc-UMR 5024/ISTEEM (CNRS), \\ Université Montpellier II, Place E. Bataillon, 34095 Montpellier Cedex 05, France \\ 3 Observatoire de Haute-Provence (CNRS), 04870 Saint-Michel-l'Observatoire, France
}

Received 19 June 2001 / Accepted 20 August 2001

\begin{abstract}
In order to study in a general manner the pulsations of the post-AGB stars we performed a grid of non-linear radiative low-mass, high-luminosity models in a wide range of $T_{\text {eff }}, L$ and $M$. Theoretical bolometric light curves and radial velocity curves were computed as well as their associated power spectra. We have also examined the behaviour of these models according to the stellar mass, the chemical composition and the opacity tables we have used in the code. Concerning the carbon rich post-AGB star HD 56126, the models best found to fit its observational characteristics are $X=0.7, Z=0.004, M=0.8 M_{\odot}, L=6000-7000 L_{\odot}$ and $T_{\text {eff }} \leq 5850 \mathrm{~K}$. Models computed with smaller mass fail to reproduce both the period and the pulsational amplitude of HD 56126, which is quite different from the pulsational amplitude of the RV Tauri stars. The previously analysed spectroscopic and photometric variations of HD 56126 appear to be consistent with a non-regular radial pulsation where the dominant pulsation mode is the first overtone. In the course of the pulsation, moderate shock waves are repeatedly generated and they propagate throughout the stellar atmosphere. They provoke a complex, asynchronous motion of the outer layers.
\end{abstract}

Key words. stars: atmospheres - stars: AGB and post-AGB - line: profiles - stars: oscillations - stars: fundamental parameters - stars: individual: HD 56126

\section{Introduction}

Over the past few years, numerous chemical and morphological studies of the circumstellar envelopes of postAGB stars (see for instance the review by Meixner 2000) have been undertaken in order to understand this brief and peculiar evolutionary stage between the end of the Asymptotic Giant Branch (AGB) and the Planetary Nebulae stage. If mass loss is well known to play an important role in the shaping and the evolution of nebulae, the phenomena of its origin are still insufficiently studied. On the other hand, both linear and non-linear modelling of radial stellar pulsation occurring in AGB stars (like Mira stars) has been undertaken (Ya'ari \& Tuchman 1996; Bessel et al. 1996). Models of dynamical atmospheres relevant to Mira stars have also been developed by Höfner \& Dorfi (1997), taking into account the well-known shock wave propagation throughout their atmosphere. Surprisingly, nowadays, while long-term observations (photometric and spectroscopic) are becoming

Send offprint requests to: A. Lèbre, e-mail: lebre@graal .univ-montp2.fr available for a few of the post-AGB stars (e.g., HD 56126, see Barthès et al. 2000), little is known about the theoretical aspects of the pulsational nature of this class of objects.

Aikawa (1991) was the first to study the properties of non-linear pulsations in post-AGB stars. Irregular oscillations with small amplitudes yielded in radial pulsation were established by means of hydrodynamic simulations. Furthermore, Aikawa \& Sreenivasan (1998) proposed that the irregular pulsations observed in the post-AGB stars could be driven by strange modes (pulsationally unstable). Non-linear models for low luminosity $\left(1500 L_{\odot}-4000 L_{\odot}\right)$ and $M=0.8 M_{\odot}$ showed that the irregularity of the pulsation increased with the luminosity, even leading to chaotic behaviour (Aikawa 1993).

Because lots of observations are available for HD 56126, a very typical carbon-rich post-AGB star, its fundamental parameters derived from observational analysis have been used to compute pulsational models. For the first time, by the means of linear and nonlinear models, Jeannin et al. (1996) could reproduce the main pulsational observed properties (period and radial 
velocity curve). The development of pulsation-induced shock waves (of relatively high amplitude) was also confirmed and even reconciled with the very small amplitude variations observed in the light curves of post-AGB stars (Kwok 1993). Furthermore, Jeannin et al. (1997) performed a theoretical spectral line analysis on few metallic elements and again compared their findings with data available for HD 56126. Turbulent motions ( of 5-8 $\mathrm{km} \mathrm{s}^{-1}$ ) were invoked to explain the observed broadening on Ba II and C I lines. Recently, new complementary and more accurate observational data of HD 56126 (both in photometry and spectroscopy) have become available (Barthès et al. 2000). These new data now provide some relevant constraints to compute non-linear pulsating models. Finally, the pulsational properties of post-AGB stars could help to identify the low-mass post-AGB stars from massive supergiants. Indeed, an analysis based only on the evolutionary status and/or morphological observations cannot make such a distinction without ambiguity. For instance, the case of the oxygen-rich star, HD 179821, is still under debate (Jura \& Werner 1999; Josselin \& Lèbre 2001; Jura et al. 2001).

In Sect. 2 we present our grids of non-linear models relevant to post-AGB stars and we examine the effects induced by different parameters such as mass or chemical composition. The opacity effect on our models is also investigated. In Sect. 3, we focus on the case of HD 56126. We then present the current observational status of this carbon-rich post-AGB star and we discuss the mass problem. Finally, conclusions are drawn in Sect. 4.

\section{Non-linear models}

\subsection{Model series}

One of the peculiarities of post-AGB stars is that they oscillate in a linearly stable mode. Indeed, as shown by Jeannin et al. (1996), the pulsational characteristics of HD 56126 correspond to the first overtone with a small amplitude. In the meanwhile, as suggested by the LNA calculations (e.g. Jeannin et al. 1996; Aikawa 1991, 1993; Zalewski 1993; Barthès et al. 2000), the fundamental mode, the first overtone, and sometimes even the second overtone are pulsationally stable in the $\mathrm{HR}$ region relevant to the post-AGB stars. Thus, only a nonlinear analysis can be used to study their pulsations and amplitudes. However, we still know little about the origin of such variability, although it is probably provoked by nonlinear modal interaction.

We performed new hydrodynamical modelling of the post-AGB stars, improving the work already undertaken by Jeannin et al. (1996). Our goal was two-fold: firstly, to study the systematic dependences of the pulsational characteristics on basic stellar parameters and on different opacities; secondly, to reconcile the presence of shock waves with the very small amplitude detected in HD 56126 (photometric variability with $\Delta V \sim 0.06-0.15 \mathrm{mag}$ ) and a dominant dynamical period of about 37 days (Barthès et al. 2000).
We used the same radiative Lagrangian code as in Jeannin et al. (1996), and varied the stellar parameters in the ranges: $M=0.6-0.8 M_{\odot}, L=4500-8000 L_{\odot}$, $T_{\text {eff }}=5600-6300 \mathrm{~K}$. These ranges correspond to the observational estimations obtained by Barthès et al. (2000).

We also tried OP (Seaton et al. 1994), OPAL92 (Rogers \& Iglesias 1992) and OPAL96 (Iglesias \& Rogers 1996) opacity tables (the numerical tables were kindly provided by Dr. I. Baraffe) corresponding to various $Z$ (0.001-0.02). The OPAL tables were supplemented with the Alexander \& Fergusson (1995) tables for low temperatures.

All models contained 90 to 110 mass zones, about one third of them being in the optically thin region (atmosphere). The initial mass density at the outer boundary was about $10^{-12} \mathrm{~g} \mathrm{~cm}^{-3}$, which allows more detailed study of the atmospheric motions. The inner boundary was chosen at $T \approx 10^{6} \mathrm{~K}$, corresponding approximately to $1-2 \%$ of the photospheric radius $R_{\mathrm{ph}}$.

The initial velocity profile corresponds to the $F$-mode with $v=1 \mathrm{~km} \mathrm{~s}^{-1}$ at the surface, which plays the role of a small disturbance in the strictly equilibrium initial model. The hydrodynamical calculation continues until the model reaches its limiting amplitude (but not always the stable limit cycle), normally after about 1000 pulsational cycles.

In Figs. 1 and 2 we present one of our extended grids of non-linear models for $X=0.7, Z=0.004$ and $M=0.8 M_{\odot}$. This series was calculated with the OPAL92 opacity table. Each model is represented for a given set $\left(L, T_{\text {eff }}\right)$, with these values corresponding to the geometrical center of each diagram. In Fig. 1, we display the computed bolometric light curves, and Fig. 2 shows their corresponding power spectra.

From these figures, systematic changes can be seen in the morphology and amplitude of the light curves, as well as in the power spectra, as $L$ and $T_{\text {eff }}$ vary. Indeed, as seen from Fig. 1, along the $L$ sequences, the amplitude increases as $T_{\text {eff }}$ decreases. Similarly, the chaotic component decreases for lower $L$ and higher $T_{\text {eff }}$ models. In some model series (e.g. for $Z=0.003, M=0.8 M_{\odot}$ with OP opacities, not shown here), the models with $L=5000 L_{\odot}$ become pulsationally stable, while the coolest models with $L=8000 L_{\odot}$ turn out to be dynamically unstable. It can also be seen from Fig. 2 that, as $T_{\text {eff }}$ diminishes, the lower modes become more enhanced for all luminosities, whilst the higher overtones diminish or even disappear. The maximum point-to-point velocity amplitude generally varies from about $10 \mathrm{~km} \mathrm{~s}^{-1}$ to $25 \mathrm{~km} \mathrm{~s}^{-1}$.

\subsection{Mass effect}

We have computed several additional grids of models with mass values $M=0.6 M_{\odot}$ and $M=0.7 M_{\odot}$, and for different chemical compositions and opacity tables. In Fig. 3 we present bolometric light curves and associated power spectra for two models with masses $0.6 M_{\odot}$ and $0.8 M_{\odot}$, having the same set of stellar parameters $\left(L=6000 L_{\odot}\right.$, 


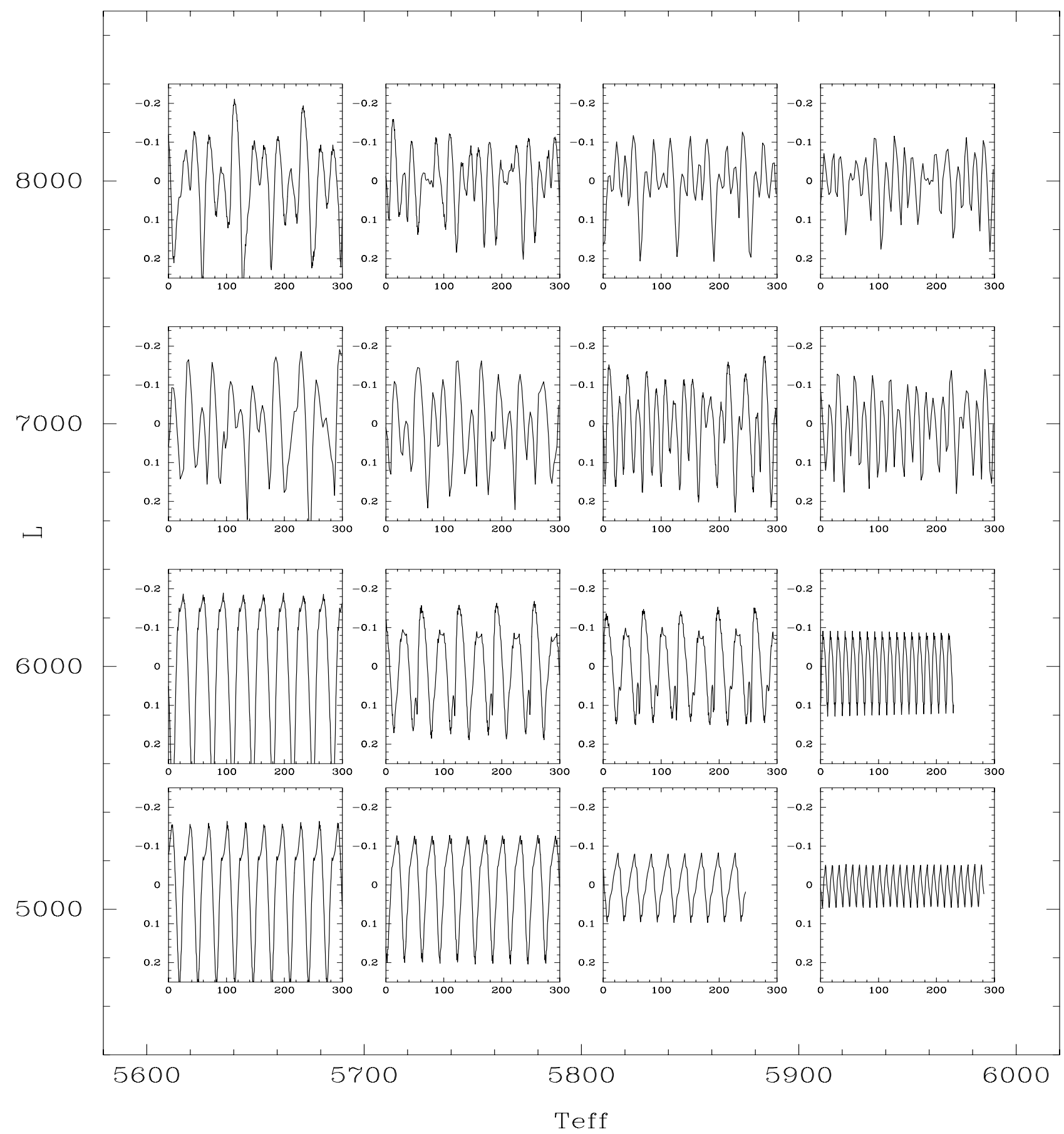

Fig. 1. Grid of computed bolometric light curves $\left(M=0.8 M_{\odot}, X=0.7, Z=0.004\right.$ and with the OPAL92 opacity table). Model axes are the time (in days) as abscissa, and $\Delta m_{\text {bol }}$ as ordinates.

$T_{\text {eff }}=5750 \mathrm{~K}, Z=0.004$ and OPAL92 opacity table). As can be seen, the amplitude drastically increases as the mass decreases, so that the amplitude of the $0.6 M_{\odot}$ model becomes far higher than observed. In addition, the period of the dominant component increases up to 54 days, which is about twice the stellar acoustic time (i.e. the time required for a sonic signal to get from the stellar surface to the center). This implies that, in the $0.6 M_{\odot}$ model, the dominant component is probably the unstable fundamental mode, which usually has a high amplitude. Similar character of changes is also found for other luminosities and temperatures. Unfortunately, we were unable to perform a rigorous LNA analysis of the modal stability in our models because of several limitations of our linear code. In the future we plan to perform such a study with one of the up-to-date LNA codes.

Although the observed light amplitudes of the postAGB stars refer to the visual magnitude and not to the bolometric one, the difference between them is not large (less than 10 per cent) for $T_{\text {eff }}=6000-8000 \mathrm{~K}$. In addition, for this range of $T_{\text {eff }}$, the amplitude $\Delta V$ even exceeds $\Delta m_{\text {bol }}$. Thus, the expected bolometric correction 


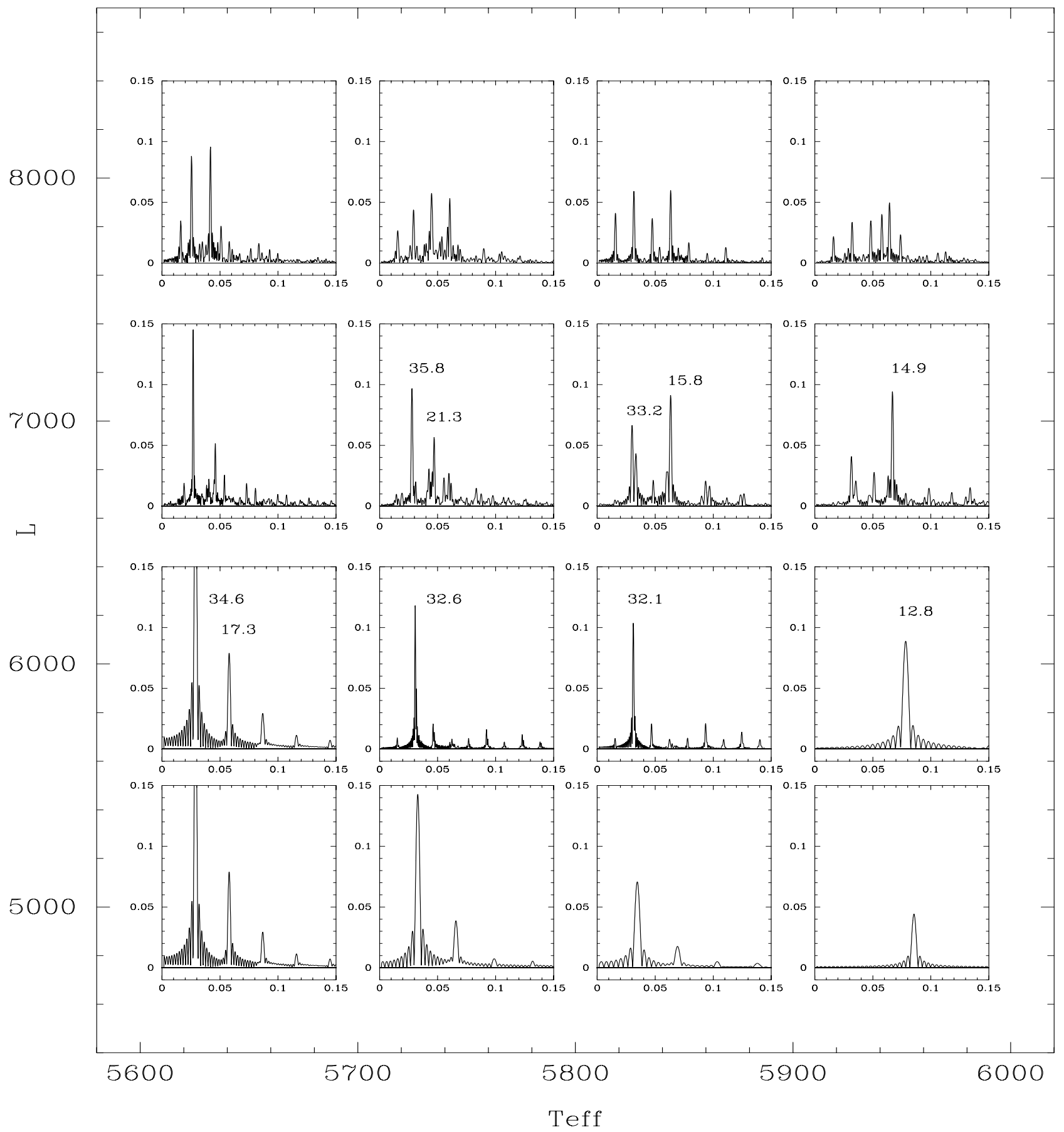

Fig. 2. Power spectra of computed light curves (same parameters as in Fig. 1). The ordinates of the graphs are the amplitude (in mag). Periods (in days) are indicated for the main components.

cannot help to overcome this theory/observation discrepancy for $0.6 M_{\odot}$ models. Also, the convective energy transport, not included in the present models, can actually reduce the instability and, as a consequence, the amplitude. It awaits to be proven by an analysis of the convective models.

So we conclude that:

- the theoretical light amplitudes of the $M=0.6 M_{\odot}$ models with the dominant periods longer than 30 days are too high $\left(\Delta m_{\text {bol }} \sim 0.45-0.5 \mathrm{mag}\right)$ with respect to the observations $(\Delta V \sim 0.06-0.15 \mathrm{mag}$ for HD 56126 , see next section);

- for $M=0.7 M_{\odot}$, the reasonable amplitudes can be obtained only for luminosities below or about $5000 L_{\odot}$, which seems too low for the post-AGB stars;

- for $M=0.8 M_{\odot}$, amplitudes of about $0.2-0.3 \mathrm{mag}$ can be obtained for luminosities up to $8000 L_{\odot}$, but light curves become more chaotic and complicated. 

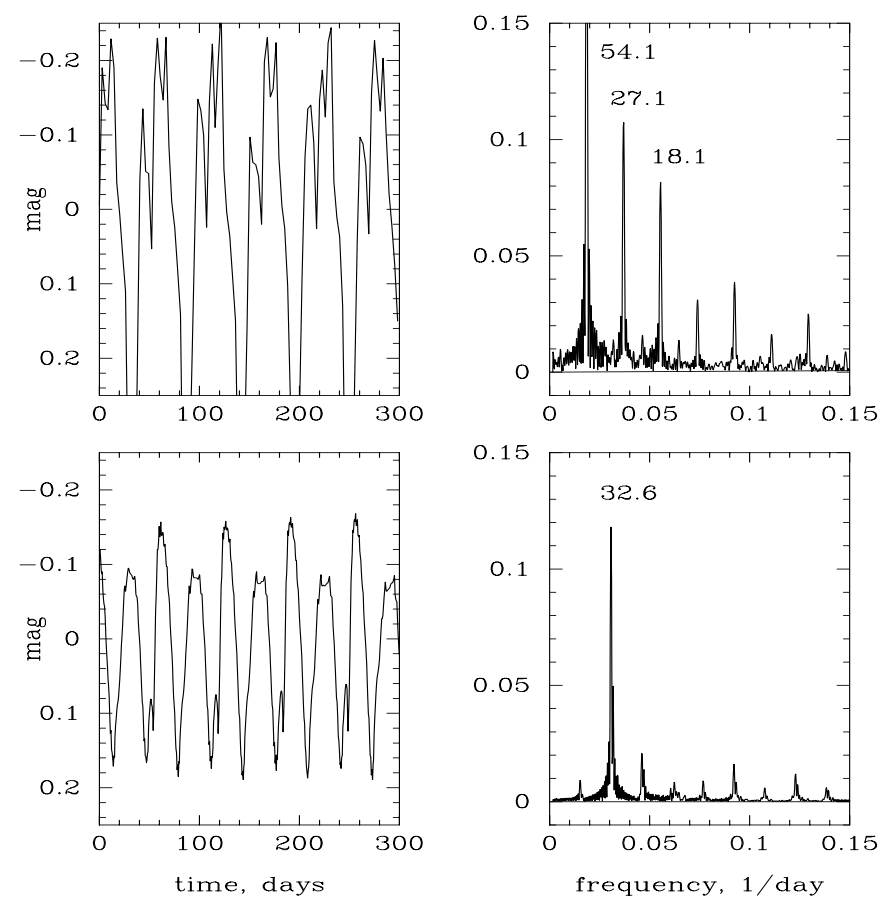

Fig. 3. Effect of stellar mass. Bolometric light curves and associated power spectra for two models computed at $L=6000 L_{\odot}$ and $T_{\text {eff }}=5750 \mathrm{~K}$ with $M=0.6 M_{\odot}$ (upper panels) and $M=0.8 M_{\odot}$ (lower panels). As in Figs. 1 and 2, the OPAL92 opacity tables were used.

\subsection{Opacity sensitivity and composition effect}

As mentioned before, a strong effect of the different opacity tables (OP, OPAL92 and OPAL96) was found. Depending on the opacity table we used, the resulting models can be quite different for the same range of parameters. Figures 4 and 5 illustrate the role of opacity for the model with $L=7000 L_{\odot}, T_{\text {eff }}=5750 \mathrm{~K}, M=0.8 M_{\odot}$ and $Z=0.001$. In Fig. 4, we compare the density and temperature distributions in the static envelopes calculated with OPAL92 (solid lines) and OP (dashed lines) versus radius. Figure 5 shows the fragments of the light curves and power spectra corresponding to these models. Despite a very small difference between the static models, the OPAL92 model has a dominant period of 36.3 days, corresponding to the first overtone, while the OP model pulsates in a higher overtone. Generally, the 36 day peak in the OP models first appears at temperatures about $100 \mathrm{~K}$ lower than in the OPAL92 models. To date, unfortunately, such a rigorous comparison is only possible for $Z=0.001$, due to a different $Z$-grid of the published tables. We note, however, that the test calculations show that the difference between the results obtained with the OP opacities for $Z=0.003$, and the OPAL92 opacities for $Z=0.004$ is larger than that between the $Z=0.001$ and $Z=0.004$ OPAL92 results.

This opacity effect poses a problem for the numerical modelling and the interpretation of the observations of individual post-AGB stars. We cannot satisfactorily explain this effect yet, although it is probably associated

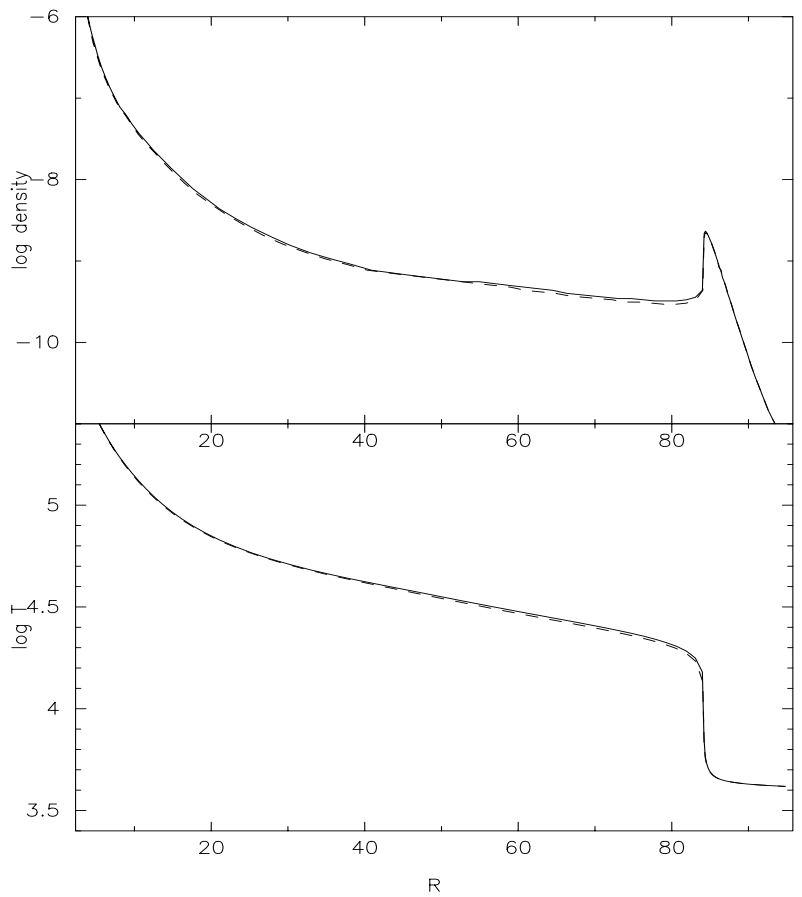

Fig. 4. Comparison of two static models with $L=7000 L_{\odot}$, $T_{\text {eff }}=5750 \mathrm{~K}, M=0.8 M_{\odot}$ and $Z=0.001$, calculated with the OPAL92 (solid lines) and OP (dashed lines) opacity tables.

with slightly different opacity gradients on $T$ or $\rho$. The LNA analysis of the modal stability could help us understand this opacity sensitivity. For the reasons mentioned above we leave this analysis to the future. However, as shown by Jeannin et al. (1996) in the OPAL92 models with $T_{\text {eff }}$ greater than $5650 \mathrm{~K}$, both the $F$-mode and the first overtone are stable. Thus, the presence of the 36.3 day peak (supposed to correspond to the first overtone) still cannot be explained. It seems that the opacity effect and the presence of the damped first overtone have the same origin, namely the nonlinear interaction between the unstable high overtones and lower stable modes.

The effect of the metal abundance is illustrated in Fig. 6. The model with $L=7000 L_{\odot}, T_{\text {eff }}=5750 \mathrm{~K}$ and $M=0.8 M_{\odot}$ has been calculated with 3 values of $Z(0.001$, 0.003 and 0.03 ) using the relevant OP opacity tables.

Two more examples of the composition effect are shown in Figs. 7 and 8 for a slightly different model: $L=6100 L_{\odot}, T_{\text {eff }}=5800 \mathrm{~K}$ and OP opacities. Figure 7 represents the results obtained with $X=0.7$ and $Z=0.001$ (upper panel) and $Z=0.003$ (lower panel). Figure 8 is similar to Fig. 7 but for $X=0.5$.

From these results we may conclude that as $Z$ increases, the amplitude also increases, with enhancement of the lower harmonics. As $X$ decreases, the amplitude somewhat diminishes, but not much.

\subsection{Beat models}

Among the regular and non-regular non-linear models we found several ones which reveal "beat" oscillation. It is 

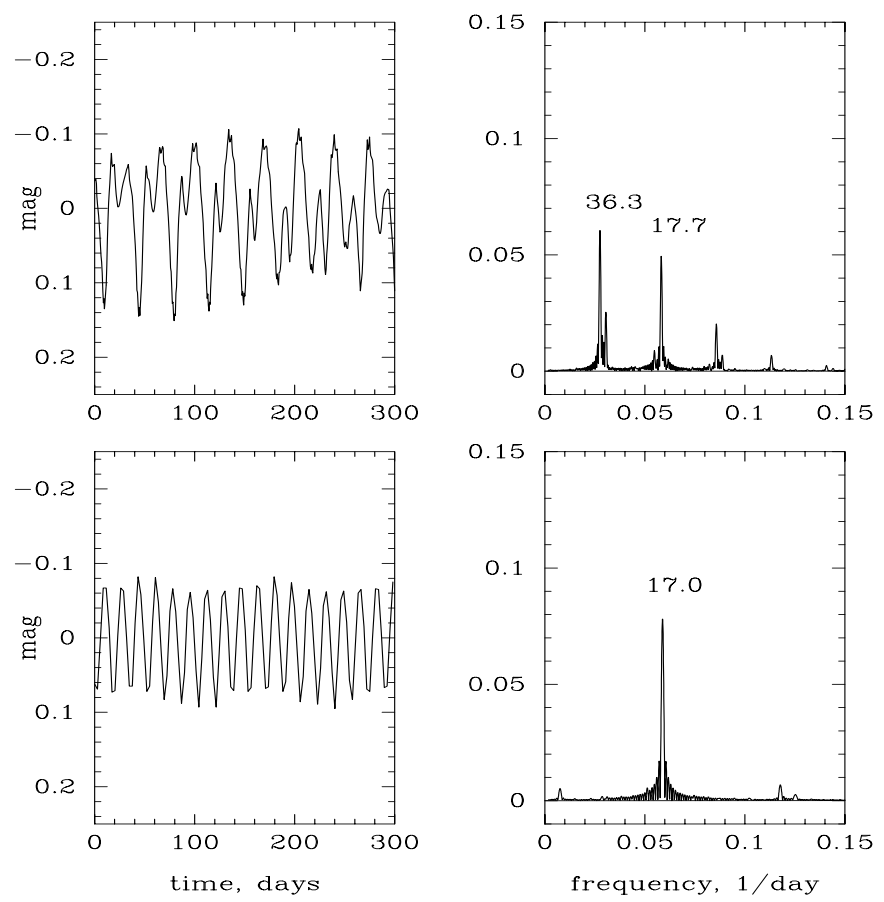

Fig. 5. Opacity sensitivity. Bolometric light curves and associated power spectra for two models computed at $L=7000 L_{\odot}$, $T_{\text {eff }}=5750 \mathrm{~K}, M=0.8 M_{\odot}$ and $Z=0.001$ with the OPAL92 (upper panels) and the OP (lower panels) opacity tables.

difficult, however, to determine the range of parameters for this phenomenon, because it depends on $Z$ as well as on the selected opacity table. We leave this point open for further study.

One of these models has been displayed in Fig. 5, upper diagram. Figure 9 shows a longer fragment of its light curve. The periodic modulation of the light curve appears stable, and the power spectrum remains the same over the integration time. A similar phenomenon was also found in the models computed with the OP and OPAL96 opacities.

As can be seen from Fig. 5, the power spectrum of the beat model is very clean and it contains only a few split peaks. It does not contain any components with a complex frequency ratio, corresponding to different pulsational modes. Thus, this effect is quite different from the well-known "beat Cepheids" phenomenon, where two linearly self-excited modes co-exist (Faulkner 1977). We suggest that this beat phenomenon in the post-AGB models has a purely nonlinear origin. In the future, more detailed studies are needed to clarify its physical nature.

\section{Application to HD 56126}

\subsection{Observational status of HD 56126}

HD 56126 (=SAO $96709=$ IRAS $07134+1005)$ is a F5Iab carbon-rich post-AGB star. A lot of observations have already been accumulated on this Pop. II object, reporting a chemical composition with a metal deficiency and an excess of $s$-elements very likely due to an efficient 3rd dredge-up (see Van Winckel \& Reyniers 2000, and
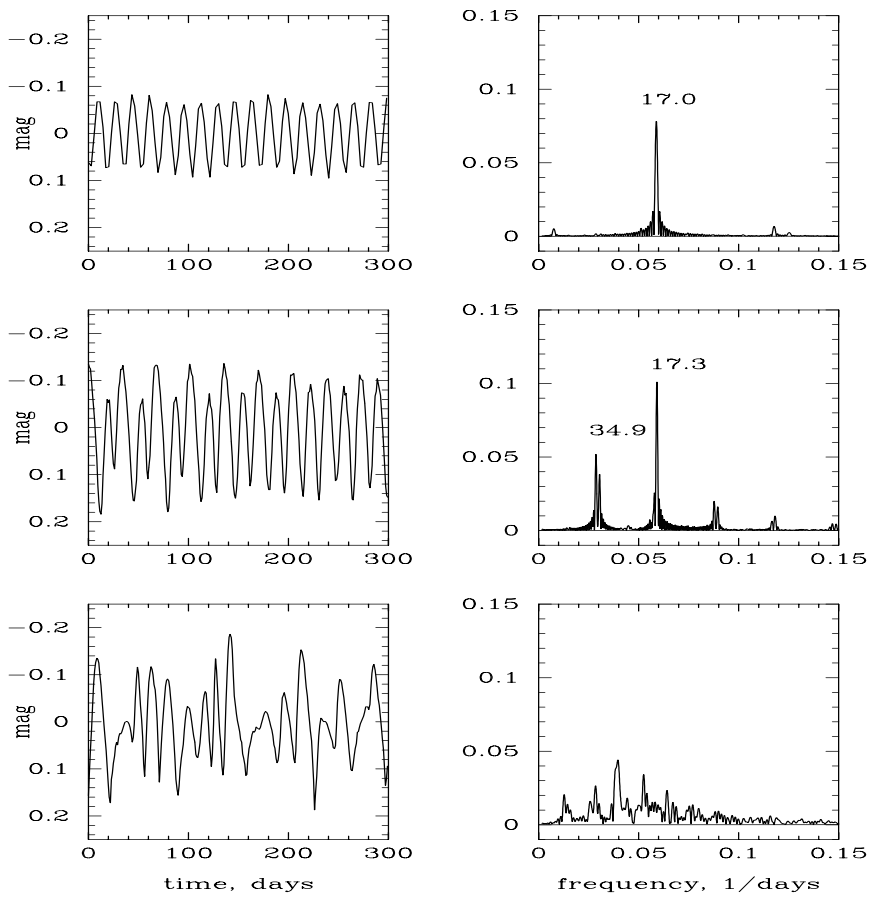

Fig. 6. Metal abundance effect on light curves (and associated power spectra). The same model as the one displayed in the lower panel of Fig. 5 is calculated with $Z=0.001,0.003$ and 0.03 (from top to bottom). OP opacities are used.
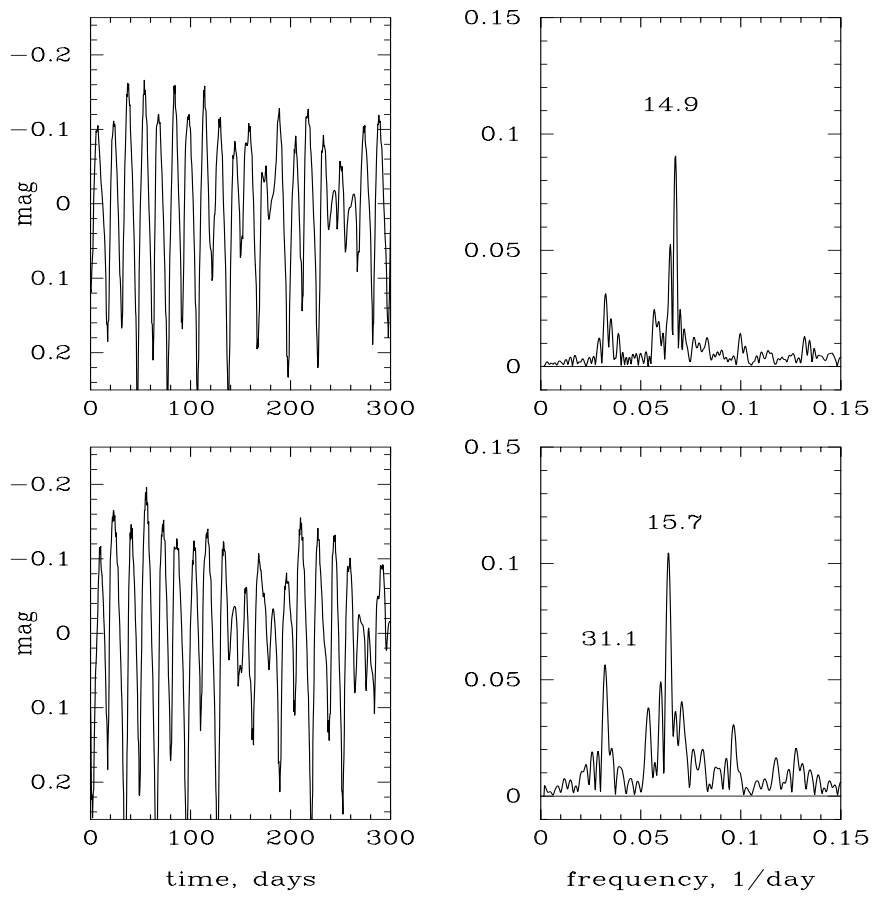

Fig. 7. Metal abundance effect for the model with $L=6100 L \odot, T_{\text {eff }}=5800 \mathrm{~K}, X=0.7$ and the OP opacities: $Z=0.001$ (upper panel) and $Z=0.003$ (lower panel).

references therein). Concerning its circumstellar features, a strong infra-red excess due to a dust shell (with a toruslike shape of the envelope) and the detection of the famous $21 \mu \mathrm{m}$ and $30 \mu \mathrm{m}$ emission features have been reported. The $21 \mu \mathrm{m}$ emission feature is attributed to 

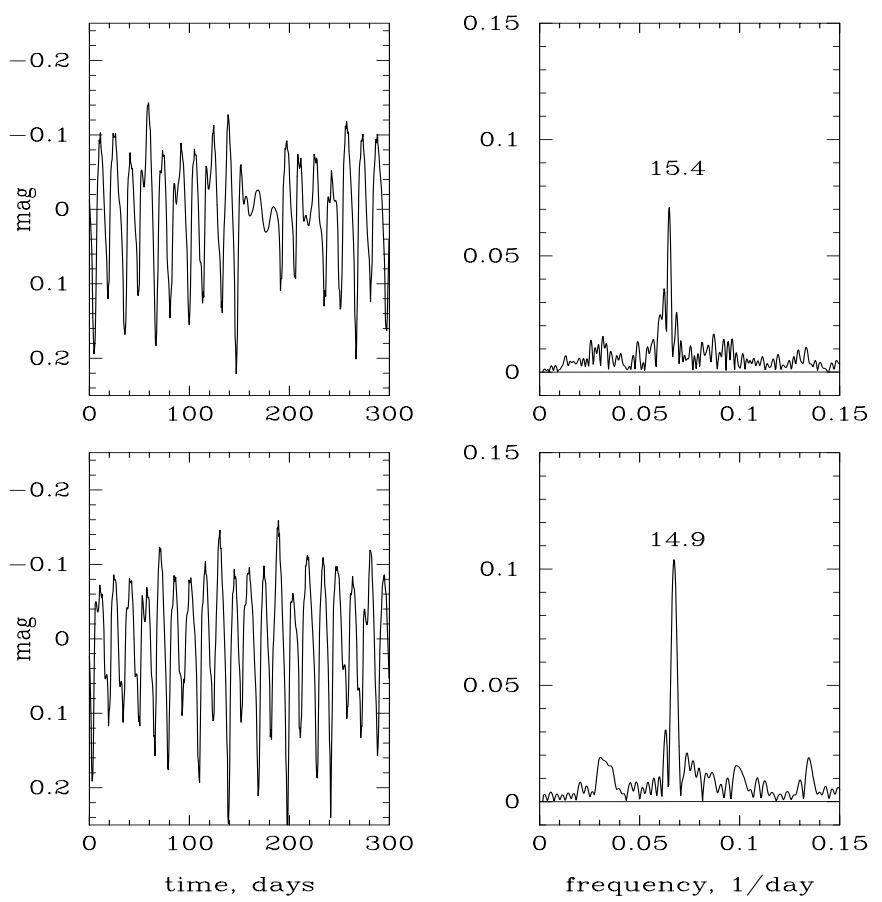

Fig. 8. Same as Fig. 7 but for $X=0.5$.

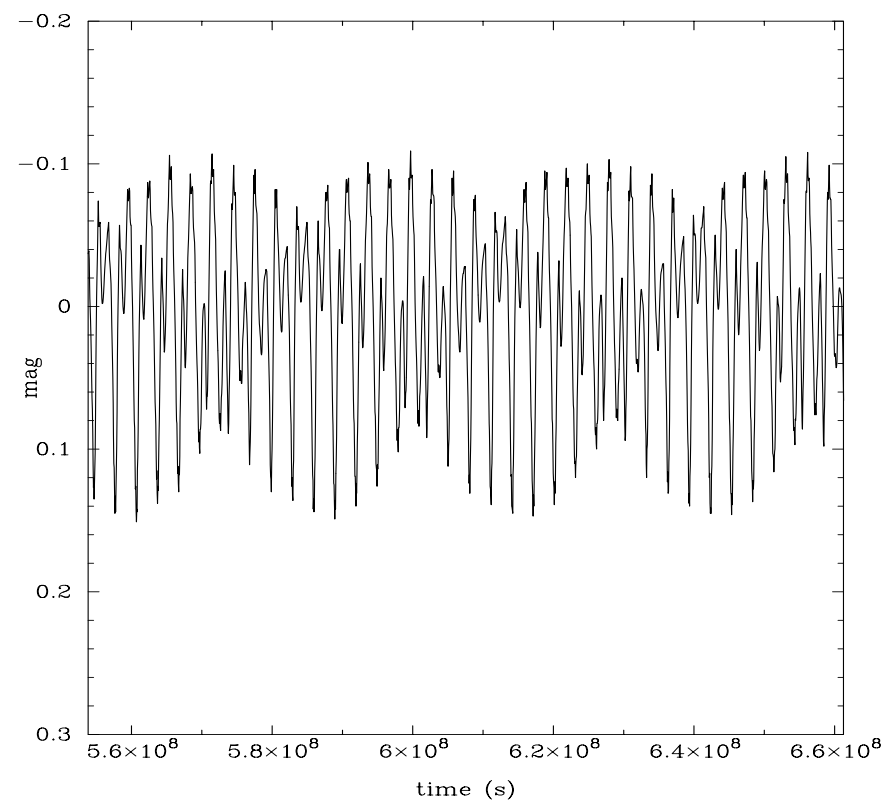

Fig. 9. Long-term light curve for the beat pulsation of the model displayed in the upper panel of Fig. 5.

titanium carbide (TiC) nanocrystals (Von Helden et al. 2000). TiC grain formation requires specific condensation conditions such as high density to provide a high enough collision rate. Because in metal-poor post-AGB stars, Ti abundance is very low (Van Winckel \& Reyniers 2000), Von Helden et al. (2000) suggested that TiC grains are formed during a short ( 100 years) phase of high mass loss rate $\left(\sim 10^{-3} M_{\odot} \mathrm{yr}^{-1}\right)$. However mid-IR images of the detached dust shell around HD 56126 (Jura et al. 2000) do not support such a violent episode.
In two previous works (Lèbre et al. 1996; Barthès et al. $2000)$, the results of two complementary surveys ${ }^{1}$ of the photospheric radial velocity (hereafter referred to as RV) have been presented. Important spectroscopic variations of the $\mathrm{H} \alpha$ and $\mathrm{H} \beta$ line profiles were established and also investigated. These variations could be significant over time intervals as short as $\sim 7$ days and the $\mathrm{H} \alpha$ line profile always exhibited one or two emission wings. For metallic lines, RV variations were reported with an amplitude $\Delta V_{\mathrm{r}} \sim 10-15 \mathrm{~km} \mathrm{~s}^{-1}$. These data suggest that strong atmospheric motions occur, possibly with shock wave propagation (amplitude $\sim 40-47 \mathrm{~km} \mathrm{~s}^{-1}$ ). On the other hand, Bogaert (1994) established the photometric variability of HD 56126, from the small and irregular variations (amplitude $\Delta V=0.06-0.15 \mathrm{mag}$ ) observed in the photometric light curve $^{2}$. A period of $\sim 40$ days was proposed from the analysis of the light curve.

For this reason and in order to determine the pulsation period(s) of HD 56126, Barthès et al. (2000) have analysed all these long-term photometric and spectroscopic data (spanning $\sim 8$ years) using spectral and CLEANbased methods. In spite of irregular sampling and seasonal gaps in both data sets, a clear main period of about $36.8 \pm 0.2$ days was found. Weaker secondary oscillations, probably related to the irregularity of the pulsation, were also detected. No prominent Fourier component was found at $\sim 2 P$ and thus the pulsation of HD 56126 is certainly quite different from that of RV Tauri variables (Tuchman et al. 1993). Barthès et al. (2000) also performed a linear non-adiabatic (LNA) analysis and the only radial linear mode that fits the period of 36.8 days is the first overtone. This LNA analysis also puts constraints on the stellar fundamental parameters of HD 56126, and taking into account the AGB core mass-luminosity relation (Boothroyd \& Sackmann 1988), the best range of data appeared to be the following: $L=5250-8160 L_{\odot}, T_{\text {eff }}=5700-6100 \mathrm{~K}$, $M=0.57-0.63 M_{\odot}$ and $\log g=0.38-0.49$. Owing to its significantly higher temperature, HD 56126 is probably more evolved than post-AGB RV Tauri stars. These new and more accurate observational constraints have been the basis for the input parameters included in the non-linear modelling we have presented in the previous section.

Although such long-term observations are now available for this object, there is still no convincing evidence that this star is a binary like several other post-AGB stars, but binarity cannot be definitely ruled out. Moreover some of the fundamental parameters of HD 56126 still suffer from a relatively large uncertainty (as $\log g, T_{\text {eff }}$ ) partly because they are intrinsically variable. In particular, the luminosity (or the distance), and the mass are not well

\footnotetext{
${ }^{1}$ Based on spectroscopic data obtained with the $1.52 \mathrm{~m} /$ AURELIE and the $1.93 \mathrm{~m} /$ ELODIE telescopes/instruments at O.H.P. and with the CAT/CES telescope/instrument at ESO/La Silla.

${ }^{2}$ Based on photometric data obtained with the $70 \mathrm{~cm}$ Swiss Telescope at ESO/La Silla.
} 
determined, and studying the pulsation properties may help to narrow the possible range of these parameters.

\subsection{A model for HD 56126}

Among all the grids we have computed, and with regard to the pulsational behaviour of HD 56126, the best radiative models for this object are found to have the following parameters: $L=6000-7000 L_{\odot}, T_{\text {eff }} \leq 5850 \mathrm{~K}, M=0.8 M_{\odot}$, $X=0.7$ and $Z=0.004$. As we have emphasized above, and as can be seen from Fig. 3, the models computed at $M=0.6 M_{\odot}$ clearly fail to reproduce the pulsational amplitudes, or dominant periods inferred for HD 56126 .

As one of the most appropriated models for HD 56126 , we have chosen the one with $L=6000 L_{\odot}, T_{\text {eff }}=5750 \mathrm{~K}$, $M=0.8 M_{\odot}$ and $Z=0.004$, computed with the OPAL92 opacities. This model presents a complex light curve with small amplitude alternations (see Fig. 1) and a main component at $P=32.6$ days (see Fig. 2). Figure 10 displays the theoretical velocity curve and its associated power spectrum. A velocity amplitude of $\sim 15 \mathrm{~km} \mathrm{~s}^{-1}$ and a main period of 32.6 days are found. This model fulfils relatively well the pulsational features we observed for HD 56126 . Moreover the $T_{\text {eff }}$ of $5750 \mathrm{~K}$ is in very good agreement with the one we derived from high resolution spectroscopy (Lèbre et al. 1996).

The power spectrum of this model is less noisy than, for example, in the $L=7000 L_{\odot}$ models, and it has several small clean components. The observed spectrum of HD 56126 seems to be more chaotic. However, as stressed by Barthès et al. (2000), these observed secondary components are probably not significant due to a lack of observational data. Hence, our choice of model was dictated mainly by the following points: relevant period and amplitude, and only one dominant spectral component.

The irregular pulsations of the post-AGB models at high luminosities were studied by Aikawa (1991, 1993), who suggested the high-dimensional nature of chaos. Although it seems that our models confirm this conclusion, we did not consider in detail this problem in the present paper.

In Fig. 11, we present its theoretical light curve (upper panel) and radius variations of different mass zones. Large atmospheric extension above the photosphere is the result of repeated propagation of the compression/shock waves. As already suggested from high resolution spectroscopic observations (Lèbre et al. 1996; Barthès et al. 2000), the shock wave propagation generates complex motions in the upper part of the atmosphere. Also, it can be clearly seen that the pulsation in the inner regions is mostly in the form of running waves due to the low density gradient in the envelope. This is also typical for RV Tauri stars, but the alternating character of their light curve is due to the excitation of different modes, i.e. to the non-linear coupling of the F-mode and $1 \mathrm{H}$ overtone (Fokin 1994).

For the same model, we also present in Fig. 12 the development and propagation of the compression/shock
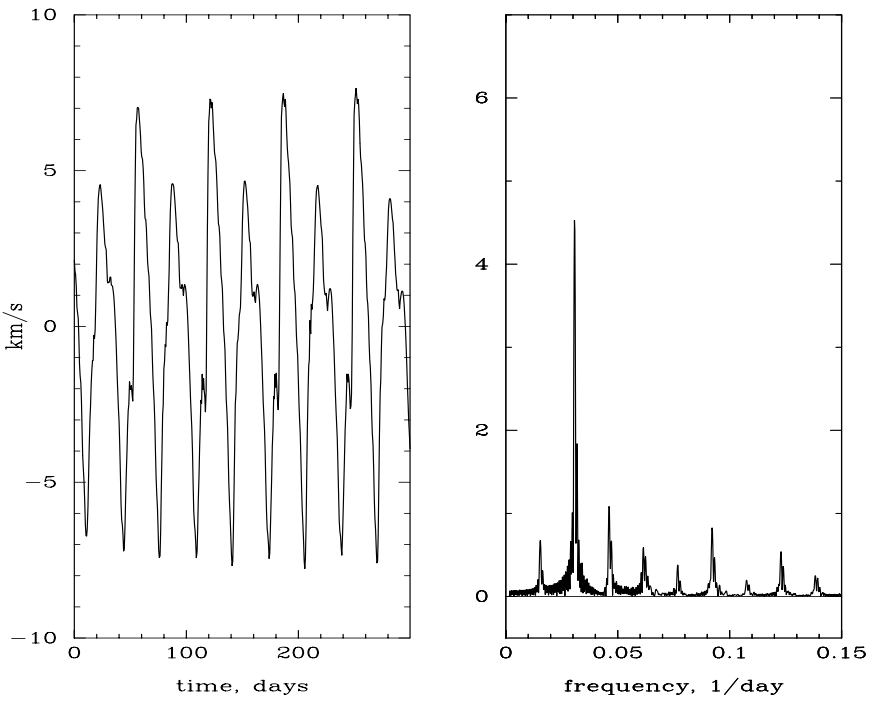

Fig. 10. Theoretical velocity curve (left panel) and associated power spectrum for a HD 56126 model computed with $L=6000 L_{\odot}, T_{\text {eff }}=5750 \mathrm{~K}, M=0.8 M_{\odot}$ and $Z=0.004$. The OPAL92 opacities were used. The ordinate of the power spectrum is in $\mathrm{km} \mathrm{s}^{-1}$.

waves throughout the stellar structure (mass grid). The strongest wave propagates outwards and reaches its maximum amplitude $\left(\sim 20 \mathrm{~km} \mathrm{~s}^{-1}\right)$ at about the 85 th mass zone (over a total of 90 zones). Note that the downward compression wave also has considerable amplitude (of about $10-15 \mathrm{~km} \mathrm{~s}^{-1}$ ) until it dissipates below the 40th zone.

Because these shock amplitudes are relatively moderate, the question is: can such a shock produce the Balmer emission observed in the real star? Although the answer can be obtained only after detailed transfer calculations and analysis of the post-shock structure, we cannot exclude this possibility. If the observed Balmer emission has a thermal origin, i.e., if it is formed in the optically thick post-shock region of radiative relaxation, then even a weak shock can - in principle - provoke the emission. In this case, the emission intensity would depend on the total cooling rate, which in turn controls the optical width of the hot shocked gas. Consequently, the correct estimation of the cooling rate becomes very important (Fokin et al. 2000). The effect of the extended atmosphere can also enhance the emission. If the Balmer transitions are controlled by photoionizations, the situation could be different, and therefore needs detailed study. We hope to investigate this problem in a forthcoming paper.

\subsection{A mass problem?}

Because non-linear pulsating models do not take into account the stellar evolution, it is important to check if the adopted luminosity and mass are consistent with the mass-luminosity $\left(M_{\text {core }}-L\right)$ relation (Paczynski 1971; Boothroyd \& Sackmann 1988). Concerning HD 56126, it may appear surprising that the best pulsational models 


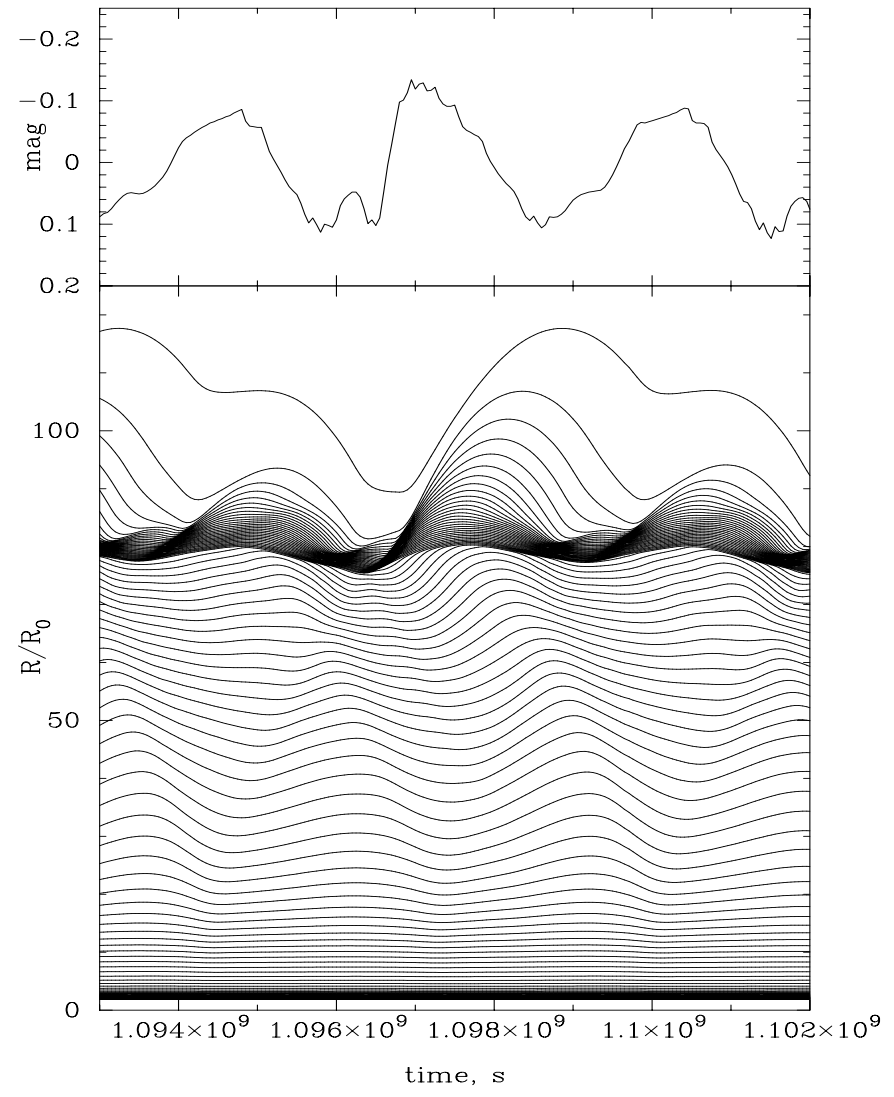

Fig. 11. Theoretical light curve (upper panel) and associated radius variation of different mass zones for a HD 56126 model computed with $L=6000 L_{\odot}, T_{\text {eff }}=5750 \mathrm{~K}, M=0.8 M_{\odot}$ and $Z=0.004$. The OPAL92 opacities were used.

are found for $L=6000-7000 L_{\odot}$ and $M=0.8 M_{\odot}$. Within this narrow range of luminosity and using Paczynski's relation (1971), the mass core would be of the order of $0.6^{-}$ $0.65 M_{\odot}$, while with $M=0.8 M_{\odot}$ the luminosity would be very large $\left(\sim 16000 L_{\odot}\right)$. The Boothroyd \& Sackmann's relation (1988) which takes into account the chemical composition and the convective mixing length, would lead to similar values. Recently, Herwig et al. (1998) have shown that the mass-luminosity relation could be violated notably by the occurrence of the third dredge-up. These authors showed that during the last thermic interpulse of the AGB-life, the luminosity (for a given mass core) can be even higher than the one deduced from Paczynski's relation. In this case, adopting $M \simeq 0.8 M_{\odot}$ would lead to a luminosity of about $20000 L_{\odot}$ (see Fig. 2 from Herwig et al. 1998). Consequently, our results (combination of $M$ and $L$ ) seem to be in contradiction to those from the mass-luminosity relations. Note that, in the Paczynski's relation, we replace the mass core, $M_{\text {core }}$, (constitued by a degenerated carbon oxygen core, surrounded by the helium shell source, inert pure helium shell and hydrogen shell sources), by the total stellar mass $M_{\text {star }}$, with $M_{\text {star }}=M_{\text {core }}+M_{\text {envelope }}$. Since $M_{\text {envelope }} \leq$ $10^{-2} M_{\odot} \quad$ (Paczynski 1971; Blöcker 1995), it is justified to replace $M_{\text {core }}$ by $M_{\text {star }}$ in the mass-luminosity relation. So, this approximation cannot explain the disagreement

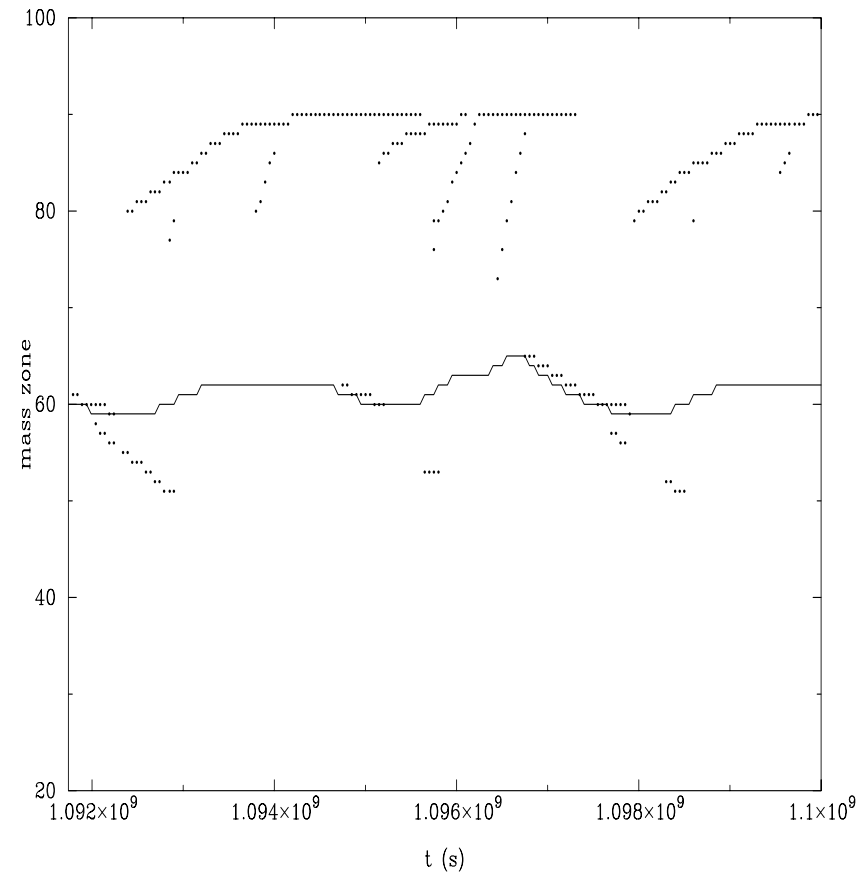

Fig. 12. Propagation of the compression and shock waves in the model shown in Fig. 11. The points indicate the position of the local maxima of compression through the Lagrangian grid.

between our results and those from the $M_{\text {core }}-L$ relations. Concerning HD 56126, the LNA analysis applied to a long period of observations (Barthès et al. 2000) led to a mass range of $M=0.57-0.63 M_{\odot}$. Such low mass values are in better agreement with the $M_{\text {core }}-L$ relation and with the evolutionary status of this metal-deficient Pop. II star.

How can such a discrepancy be explained? Our preference for the $0.8 M_{\odot}$ models has been dictated by the light amplitude selection. However, even for the best models, this amplitude still remains higher than observed (more than $0.2 \mathrm{mag}$ instead of $0.06-0.15 \mathrm{mag}$ ). On the contrary, the velocity amplitudes are in better agreement with observations even for low mass models. Because the velocity calculation is usually more correct than that of the light curve, this can imply that our flux calculation is not precise enough. Indeed, in Lagrangian codes the photospheric zones are not resolved enough, so that the precision of the temperature gradient estimation there is low. New models using an adaptative grid code can certainly avoid this disadvantage As well, we should keep in mind that our code is a radiative one, whereas the models with $P=30$ 40 days are located in the red part of the considered $T_{\text {eff }}$ range, where convection can be efficient. Convection can equally diminish the light amplitude and, possibly, even change the pulsational behaviour of the model. Because of the very complicated nonlinear nature of oscillations of the post-AGB stars, we must consider the present results cautiously.

\section{Conclusion}

We have computed grids of non-linear radiative models (theoretical light and radial velocity curves, and respective 
associated power spectra) for a wide range of $T_{\text {eff }}, L$ and $M$ relevant to the post-AGB stars.

For the pulsational behaviour of these post-AGB models, a strong dependence on the opacities was found. In this study we tested OP, OPAL92 and OPAL96 opacity tables, and we obtained quite different dynamical behaviour for the same basic stellar parameters. This suggests that the opacity problem still exists despite the recent obvious progress in the opacity projects. It seems possible that this phenomenon is closely related to the main feature of the post-AGB models, i.e. to the fact that they pulsate predominantly on a linearly dumped first overtone. We hope that future LNA analysis, together with the nonlinear study of the modal interaction, will clarify this point. Several beat models were found in the present survey. Their pulsations reveal very clean power spectra with split peaks, which implies that this phenomenon has a non-linear nature, although it is not well understood as yet.

Concerning the carbon rich post-AGB star HD 56126, we find that, among all the grids we have computed with the OPAL92 opacities, the best radiative models fitting its observational characteristics are found to have $X=0.7, Z=0.004, M=0.8 M_{\odot}, L=6000-7000 L_{\odot}$ and $T_{\text {eff }} \leq 5850 \mathrm{~K}$. The models computed with smaller masses $\left(0.6-0.7 M_{\odot}\right)$ reveal much higher bolometric amplitudes (of about $0.45-0.5 \mathrm{mag}$ ) and clearly fail to reproduce the light curve of HD 56126. On the contrary, the velocity amplitudes $\left(10-25 \mathrm{~km} \mathrm{~s}^{-1}\right)$ are in agreement with the observations, even with the low-mass models.

The mass of $0.8 M_{\odot}$, however, is in contradiction to the current evolutionary theory, which predicts masses smaller than $0.65 M_{\odot}$ for the luminosity range of $4000-8000 L_{\odot}$. Such a discrepancy may partly be due to the disadvantages of the radiative code we used, or to the unavoidably poor resolution in the hydrogen ionization zone in the Lagrangian zoning. Test calculations with non-radiative code would be helpful to clarify this point.

Another explanation concerns the temperature range we have adopted as input parameters of the models. This temperature range $(5600 \mathrm{~K}$ to $6300 \mathrm{~K})$ results from the LNA analysis and from the long-term observations of HD 56126 (Barthès et al. 2000). For this star, Jeannin et al. (1996) also obtained an effective temperature of $T_{\text {eff }}=5750 \mathrm{~K}$ by comparing $\mathrm{H} \alpha$ profiles with synthetic spectra. However, higher temperatures have also been reported for HD 56126. By modelling the IR flux distribution, Van der Veen et al. (1989) have found $T_{\text {eff }}=6600 \mathrm{~K}$ and Van Winckel \& Reyniers (2000) derived $T_{\text {eff }}=7250 \mathrm{~K}$ from the analysis of a large number of Fe lines (in the visible spectrum). Thus, the temperature of HD 56126 may fall outside the range we have adopted for the present work, and it may be possible to find some less massive models with $L=5000-8000 L_{\odot}$ and $T_{\text {eff }}>6500 \mathrm{~K}$ which would be in agreement with the observations and with the stellar evolution as well. We intend to study these "hot" models in a forthcoming paper.
Acknowledgements. The authors are grateful to Dr. I. Baraffe who kindly provided them with the OPAL opacity tables, to Dr. D. Barthès for helpful discussion and to the referee of this paper (Dr. T. Aikawa) for his valuable comments. Jason Canham is thanked for a careful reading of the text. A. B. F. also acknowledges the hospitality of the GRAAL group at the Université of Montpellier and the financial support from the CNRS during his 3-month stay in France.

\section{References}

Aikawa, T. 1991, ApJ, 374, 700

Aikawa, T. 1993, MNRAS, 262, 893

Aikawa, T., \& Sreenivasan, Sr. 1998, ASP Conf. Ser. 135, ed. P. A. Bradley, \& J. A. Guzik, 234

Alexander, D. R., \& Ferguson, J. W. 1995, ApJ, 437, 879

Barthès, D., Lèbre, A., Gillet, D., \& Mauron, N. 2000, A\&A, 359,168

Bessell, M. S., Scholz, M., \& Wood, P. R. 1996, A\&A, 307, 481

Blöcker, T. 1995, A\&A, 297, 727

Bogaert, E. 1994, Ph.D. Thesis, Leuven University, Belgium

Boothroyd, A. I., \& Sackmann, I. J. 1988, ApJ, 328, 641

Fokin, A.B. 1994, A\&A, 292, 133

Fokin, A. B., Massacrier, G., \& Gillet, D. 2000, A\&A, 355, 66

Faulkner, D. J. 1977, PAS Austral., 3, 124

Herwig, F., Schönberner, D., \& Blöcker, T. 1998, A\&A, 340, L43

Höfner, S., \& Dorfi, E. A. 1997, A\&A, 319, 648

Iglesias, C. A., \& Rogers, F. J. 1996, ApJ, 464, 943

Jeannin, L., Fokin, A. B., Gillet, D., \& Baraffe, I. 1996, A\&A, 314, L1

Jeannin, L., Fokin, A. B., Gillet, D., \& Baraffe, I. 1997, A\&A, 326,203

Josselin, E., \& Lèbre, A. 2001, A\&A, 367, 826

Jura, M., Velusamy, T., \& Werner, M. W. 2001, ApJ, in press

Jura, M., Chen, C., \& Werner, M. W. 2000, ApJ, 544, L141

Jura, M., \& Werner, M. W. 1999, ApJ, 525, L113

Klochkova, V. G. 1995, MNRAS, 272, 710

Kwok, S. 1993, ARA\&A, 31, 63

Lèbre, A., Mauron, N., Gillet, D., \& Barthès, D. 1996, A\&A, 310,923

Meixner, M., Skinner, C., Graham, J., et al. 1997, ApJ, 482, 897

Meixner, M. 2000, ASP Conf. Ser. 199, ed. J. H. Kastner, N. Soker, \& S. Rappaport, 135

Paczynski, B. 1971, Acta Astron., 21(4), 417

Rogers, F. J., \& Iglesias, C. A. 1992, ApJS, 79, 507

Parthasarathy, M., Garcia-Lario, P., \& Pottasch, S. R. 1992, A\&A, 264, 159

Seaton, M. J., Yan, Y., Mihalas, D., \& Pradhan, A. K. 1994, MNRAS, 266, 805

Tuchman, Y., Lèbre, A., Mennessier, M-O., \& Ya'ari, A. 1993, A\&A, 271, 501

Van der Veen, W. E. C. J., Habing, H. J., \& Geballe, T. R. 1989, A\&A, 226, 108

Van Winckel, H., \& Reyniers, M. 2000, A\&A, 354, 135

Von Helden, G., Tielens, A. G. G. M., Van Heijnsberger, D., et al. 2000, Science, 288, 313

Ya'ari, A., \& Tuchman, Y. 1996, ApJ, 456, 350

Zalewski, J. 1993, Acta Astron., 43, 431 